\title{
Niveles de hemoglobina y su relación con la ingesta de proteínas de alto valor biológico y hierro en gestantes
}

\section{Hemoglobin levels and its relationship with the intake of high biological value protein and iron in pregnancy}

\author{
Mario Echenique-Yupanqui ${ }^{1}$ iD, Sissy Espinoza-Bernardo² ${ }^{\text {iD }}$ \\ 1. Hospital de Chincha del Seguro Social de Salud-EsSalud. Ica, Perú. Correo: mmeeyy24@hotmail.com - https://orcid.org/0000-0001-5915-1493 \\ 2. Universidad Nacional Mayor de San Marcos. Lima, Perú. Correo: sespinozab@unmsm.edu.pe - https://orcid.org/0000-0001-9413-7320 \\ Tipología: Artículo de investigación científica y tecnológica \\ Para citar este artículo: Echenique-Yupanqui M, Espinoza-Bernardo S. Ingesta de proteínas de alto valor biológico y hierro con niveles de hemoglobina en gestantes. \\ Duazary. 2020 julio - septiembre; 17(3): 43 - 53. Doi: http://dx.doi.org/10.21676/2389783X.3321 \\ Recibido en junio 26 de 2019 \\ Aceptado en septiembre 26 de 2019 \\ Publicado en línea en abril 18 de 2020
}

Palabras clave: hemoglobina; proteínas; hierro; mujeres embarazadas.

Keywords: Hemoglobin; Protein; Iron; Pregnant women.

\section{RESUMEN}

En el Perú la anemia en gestantes es de 29,6\%, siendo un indicador de malnutrición y de problemas de salud. Una dieta con nutrientes en calidad, cantidad y proporción adecuada puede reducir las complicaciones durante el embarazo. El objetivo fue determinar los niveles de hemoglobina y su relación con la ingesta de proteínas de alto valor biológico y hierro en gestantes que acuden al Hospital de Chincha de EsSalud. Estudio analítico, transversal y correlacional. Muestreo aleatorio simple conformado por 104 gestantes que se atendieron por consulta externa en el Servicio de Nutrición del Hospital de Chincha de EsSalud. Los datos fueron recolectados por una entrevista y una encuesta de frecuencia de consumo de alimentos. El estadístico empleado fue el análisis de regresión lineal bivariado y múltiple. Se encontró 35\% de gestantes anémicas, $48 \%$ y $59 \%$ de gestantes con ingesta deficiente de proteínas de alto valor biológico y hierro, respectivamente. Hubo correlación alta y estadísticamente significativa entre los niveles de hemoglobina con la ingesta de proteínas de alto valor biológico y hierro $\left(r=0,88 ; r^{2}=0,78 ; p<0,001\right)$. Se concluyó que los niveles de hemoglobina tienen relación directa y fuerte con la ingesta de proteínas de alto valor biológico y hierro en gestantes.

\section{ABSTRACT}

In Peru, 29.6\% of pregnant women have anemia, a clear indicator of malnutrition and health issues. A highly nutritious diet can reduce complications during pregnancy. The objective was to determine the levels of hemoglobin and its relationship with the intake of high biological value protein and iron in pregnant women who treat themselves at Chincha Hospital - ESSALUD. Analytical, cross-sectional and correlational study. Simple, random sampling consisting of 104 pregnant women who were treated through outpatient consultation at the nutritional ward of Chincha Hospital - ESSALUD. The data was collected through an interview and a survey about the frequency of food consumption. The statistic used was the bivariate and multiple linear regression analysis. In the results it was found that $35 \%$ of the women had anemia, $48 \%$ had low intake of high biological value protein and $59 \%$ had low intake of iron. There was a high and statistically significant correlation between hemoglobin levels and the intake of high biological value protein and iron $(r-0,88 ; r 2-0,78 ; p<0,001)$. It was concluded that hemoglobin levels have a direct and strong relationship with the intake of high biological value protein and iron in pregnant women. 


\section{INTRODUCCIÓN}

Los bajos niveles de hemoglobina $(\mathrm{Hb})$ se definen como su concentración en sangre por debajo de los valores normales. La anemia es una alteración de la sangre caracterizada por la disminución de la concentración de hemoglobina a un rango menor al establecido ${ }^{1}$. La Organización Mundial de la Salud (OMS) considera que para que exista anemia durante el embarazo, los valores de $\mathrm{Hb}$ deben ser menores de $11 \mathrm{~g} / \mathrm{dL}^{2}$ y la clasifica en anemia leve (Hb: 10-10,9 g/dL), moderada ( $\mathrm{Hb}$ : 79,9 $\mathrm{g} / \mathrm{dL})$ y severa $(\mathrm{Hb}<7 \mathrm{~g} / \mathrm{dL})^{2,3}$, siendo además un indicador de malnutrición y problemas de salud.

La anemia es la más frecuente de las enfermedades que pueden coincidir con el embarazo o ser provocadas por este ${ }^{4}$. Una gestante con valores de $\mathrm{Hb}<11 \mathrm{~g} / \mathrm{dL}$ tiene alto riesgo de presentar complicaciones hemorrágicas, tasas elevadas de parto pretérmino, niños con bajo peso al nacer y mayor número de enfermedades neonatales ${ }^{3,5}$. Los bajos niveles de hemoglobina afectan a cerca de la mitad de todas las embarazadas en el mundo. De acuerdo a la OMS la prevalencia mundial de anemia en gestantes es casi $42 \%$, para América Latina y El Caribe la prevalencia oscila entre 20 y $39 \%^{2}$. En el Perú, de acuerdo al ENDES 2017, la anemia en gestantes es de $29,6 \%^{3}$, cifra que disminuye con la edad materna, pero aumenta con la edad gestacional.

Durante el embarazo suceden cambios anatómicos y fisiológicos que afectan todas las funciones de la gestante. La importancia de la alimentación y nutrición en la etapa reproductiva de toda mujer es indiscutible, el crecimiento fetal durante el embarazo es un proceso que requiere del aporte constante de nutrientes. Una dieta de calidad es aquella que satisface las necesidades nutricionales de la gestante, incluye la energía y todos los nutrientes como carbohidratos, proteínas, grasas, vitaminas y minerales, que se ingiere en cantidades apropiadas $y$ en proporciones idóneas entre sí. Una gestante con una dieta que aporte los nutrientes en cantidad y calidad tiene mayores probabilidades de no presentar complicaciones durante el embarazo y el parto, además de tener un hijo saludable ${ }^{7}$. Las complicaciones del embarazo asociados a déficit nutricional incluyen anemia, hemorragia materna, preeclampsia y eclampsia, retardo del crecimiento intra uterino, defectos del tubo neural, parto pretérmino, alteración en el peso materno y del recién nacido ${ }^{6,8,9}$.

Debido al incremento de necesidades de hierro, la anemia ferropénica es la enfermedad hematológica de mayor prevalencia en la embarazada ${ }^{10}$; no obstante, la síntesis de hemoglobina requiere de muchas sustancias, pero las deficiencias que tienen más probabilidad de afectarla son hierro, cobalamina y folato, nutrientes que están contenidos principalmente en proteínas de origen animal ${ }^{2}$. En este sentido, el estado nutricional adecuado durante el embarazo puede reducir complicaciones perinatales, contribuyendo a la reducción de morbimortalidad materno-infantil ${ }^{11}$.

La Ingesta Dietética de Referencia (IDR) de hierro en gestantes es $27 \mathrm{mg} / \mathrm{día}^{12}$. El tipo de hierro ingerido en los alimentos puede ser de alta o baja biodisponibilidad, el cual determinará su grado de absorción a nivel intestinal. El hierro de alta biodisponibilidad está presente en los alimentos de origen animal como carnes, pescado $y$ vísceras; su absorción intestinal varía de 15 a $40 \%$. El hierro de baja biodisponibilidad se encuentra en alimentos de origen vegetal, su absorción en el intestino es de 2 a 20\%; esta absorción dependerá de la presencia de ciertos compuestos en la dieta como fitatos/oxalatos y ácido ascórbico que disminuirán o potenciarán su absorción, respectivamente ${ }^{6}$; además, para lograr una buena absorción intestinal de hierro, es necesario de un ambiente ácido en la dieta y de las proteínas; por tanto, un consumo deficiente de ésta puede disminuir la absorción de hierro ${ }^{13}$,

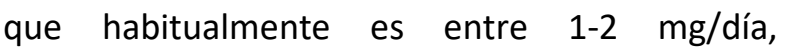
alcanzando valores de $3-5 \mathrm{mg} / \mathrm{dí}^{6,14}$.

La proteína resalta su importancia en la dieta debido a su capacidad de aportar aminoácidos para atender al mantenimiento de la proteína corporal y al incremento durante el crecimiento en las distintas etapas de vida ${ }^{15}$. Cuando esta contiene aminoácidos esenciales en cantidad adecuada se le denomina de alto valor biológico, que serán necesarias para satisfacer las demandas de nitrógeno para el crecimiento, la síntesis, y reparación tisular ${ }^{16}$. Las proteínas de origen animal son consideradas de mayor valor biológico por contener todos los aminoácidos esenciales para la síntesis proteica, en cambio las proteínas de origen vegetal tienen aminoácidos limitantes. 
Durante el embarazo se encuentra una alta incidencia de anemia debido a que es muy frecuente que se inicie esta etapa con bajas reservas de hierro debido a la pérdida de sangre por el flujo menstrual, aunado a una ingesta deficiente en hierro y proteínas ${ }^{1}$. La ingesta de proteína es derivada de la dieta, considerándose como ingesta deficiente cuando el porcentaje de adecuación es menor a 90\%; en este sentido, el porcentaje de adecuación es la relación porcentual entre la ingesta de un nutriente con la Ingesta Dietética de Referencia ${ }^{17}$.

El Comité de Nutrición y Alimentos del Instituto de Medicina de los Estados Unidos y Canadá (FNB-OIM) establece $71 \mathrm{~g}$ de IDR de proteínas en gestantes $^{6,12}$. En general, se establece que entre el 10 y $15 \%$ del valor energético total de la dieta provenga de las proteínas. Una alimentación adecuada debe aportar aproximadamente el 50\% de proteínas de alto valor biológico ${ }^{18}$. Dada a la gran variabilidad de las necesidades y de la dieta diaria, se recomienda un rango de normalidad de adecuación de $90-110 \%^{17}$.

En la dieta es importante consumir cantidades adecuadas de proteínas y hierro para prevenir problemas asociados con la ingesta deficiente que pueda devenir en anemia durante el embarazo. La aplicación de este estudio contribuirá en proponer programas de prevención e intervención en aspectos relacionados a la alimentación y nutrición que mejoren la salud de las gestantes y reduzcan su predisposición a la anemia.

El objetivo de este estudio fue determinar los niveles de hemoglobina y su relación con la ingesta de proteínas de alto valor biológico y hierro dietario en gestantes que acuden al Hospital de Chincha de EsSalud en el año 2018.

\section{MATERIALES Y MÉTODOS}

\section{Tipo de Investigación}

Se realizó un estudio analítico con datos de corte transversal y correlacional en una población de gestantes que se atendieron por consulta externa en el Servicio de Nutrición en el Hospital de Chincha del Seguro Social de Salud, EsSalud, departamento de Ica, Perú, durante los meses de abril a junio del 2018.

\section{Población y muestra}

Se realizó un muestreo aleatorio simple en una población de 142 gestantes que se encontraron en el segundo o tercer trimestre de gestación, empleando la fórmula para estudios de tipo cuantitativo para población finita, con un nivel de confianza de $95 \%$, una precisión de $5 \%^{19}$ y una desviación estándar de $0,5^{20}$. La muestra obtenida fue de 104 gestantes, clínicamente sanas, con análisis de concentración de hemoglobina en sangre, con feto único, nuevas a la consulta nutricional durante el período de gestación, suplementadas con sulfato ferroso de acuerdo a la Norma Técnica del Ministerio de Salud $^{21}$, con consumo energético promedio, mayores de 18 años y menores de 40 años.

El consumo energético promedio corresponde a la ingesta calórica adecuada, de acuerdo a la Guía Técnica de Requerimientos de Energía para la Población Peruana del Ministerio de Salud ${ }^{22}$. El consumo de energía se obtuvo de un subgrupo de gestantes elegidas aleatoriamente en el $20 \%$ de la muestra estudiada, siendo esta submuestra de 21 gestantes.

\section{Procedimientos e instrumento}

El método empleado para obtener la información de la ingesta alimentaria de la gestante fue la entrevista, y se aplicó una encuesta semicuantitativa de frecuencia de consumo de alimentos con 58 preguntas, en el cual se elaboró un listado de alimentos considerando el aporte de proteínas de alto valor biológico y hierro dietario con frecuencias de consumo diario, semanal y mensual ${ }^{23}$. El instrumento empleado fue previamente validado a través de Juicio de Expertos; para calcular estadísticamente la validez del instrumento se utilizó al Coeficiente de Proporción de Rangos (CPR), sugerido por Hernández ${ }^{24}$, obteniendo un CPR de 0,95 , lo que significa que el instrumento tuvo una validez y concordancia alta. Estas encuestas se aplicaron a aquellas gestantes que estuvieron de acuerdo en formar parte del presente estudio y que se plasmó en un formulario como soporte documental, el cual garantizó la aceptación o rechazo a la proposición (Consentimiento Informado). La entrevista a las participantes fue realizada en el área de consulta externa en el Servicio de Nutrición. Asimismo, para la obtención de información de los antecedentes clínicos de las gestantes seleccionadas, se realizó 
la revisión de historias clínicas, a fin de obtener datos relevantes al estudio que permitan cumplir con los criterios de inclusión previamente descritos.

\section{Análisis estadístico}

Para la descripción de las características de la muestra se aplicaron frecuencias, porcentajes e intervalos de confianza. Para las variables cuantitativas se aplicaron medidas de tendencia central (media) y de dispersión (desviación estándar e intervalos de confianza); además se aplicó la prueba de normalidad de Kolmogorov Smirnov. Para determinar la relación, tendencia y dispersión de las variables se utilizó la correlación de Pearson. Para el análisis se utilizó el modelo de regresión lineal bivariado y múltiple. Se utilizaron los programas estadísticos IBM SPSS Statistics versión 24.0, PAST versión 2.17 (Paleontological statistics software).

\section{Declaración sobre aspectos éticos}

Para la realización de este trabajo, se obtuvo el consentimiento informado de cada gestante, asegurando su compatibilidad con los valores, intereses y respeto, teniendo en cuenta la
Declaración de Helsinki. El proyecto de investigación fue revisado y aprobado por el Comité de Ética del Hospital de Chincha del Seguro Social de Salud, Ica, Perú (Carta No016OC-HII-RTG-GRA-ESSALUD-2018).

\section{RESULTADOS}

El total de gestantes evaluadas fueron 104 , cuyas edades fluctuaban entre 19 a 34 años, con un promedio de edad de $27,9 \pm 5,17$ años (IC $95 \%$ : $26,9-28,9)$. De este grupo, el $87,5 \%(n=91)$ tenían edades entre los 19 a 34 años (con media y

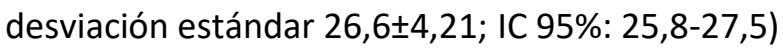
$y$ el $12,5 \%(n=13)$ tenían edades mayores a 34 años (con media y desviación estándar 36,6 $6 \pm 1,39$; IC 95\%: 35,8-37,5); el 61,5\% estaban casadas, casi el $63 \%$ presentan grado de instrucción secundaria, el 53,8\% era ama de casa, cerca del $40 \%$ fue secundigesta y multigesta respectivamente y casi el $53 \%$ de se encontraron en el tercer trimestre de gestación. Esta información se detalla en la Tabla 1.

Tabla 1: Características generales de las gestantes - 2018. Frecuencias y porcentajes.

\begin{tabular}{|c|c|c|}
\hline Características generales & $\mathbf{n}$ & $\mathbf{\%}$ \\
\hline Edad & & 81 \\
\hline $19-34$ años & 13 & 12,5 \\
\hline$\geq 35$ años & & \\
\hline Estado civil & 64 & 61,5 \\
\hline Casada & 40 & 38,5 \\
\hline Conviviente & & \\
\hline Grado de instrucción & 3 & 2,9 \\
\hline Primaria & 65 & 62,5 \\
\hline Secundaria & 36 & 34,6 \\
\hline Superior & & \\
\hline Ocupación & 56 & 53,8 \\
\hline Ama de casa & 48 & 46,2 \\
\hline Empleada & & \\
\hline Número de gestación & 22 & 21,2 \\
\hline Primigesta & 41 & 39,4 \\
\hline Secundigesta & 41 & 39,4 \\
\hline Multigesta & & \\
\hline Trimestre de gestación & 49 & 47,1 \\
\hline Segundo & 55 & 52,9 \\
\hline Tercer & 104 & 100,0 \\
\hline Total & & \\
\hline
\end{tabular}


En los niveles de hemoglobina, el $34,6 \%$ de gestantes presentaron algún grado de anemia, siendo el tercer trimestre donde se encontró la mayor frecuencia con un 43,7\%, además la anemia leve fue la más frecuente con $27,3 \%$. En la ingesta de proteínas de alto valor biológico, se observa que más de la mitad de gestantes tuvieron una ingesta normal $(51,9 \%)$, por el contrario, el tercer trimestre de gestación resalta una mayor frecuencia de ingesta deficiente de proteínas con $56,4 \%$. En la ingesta de hierro dietario, hubo una mayor frecuencia de ingesta deficiente en gestantes con 58,7\%, esta tendencia también se observó en el tercer trimestre de gestación donde la ingesta deficiente fue de $67,3 \%$. Los datos señalados en este párrafo se muestran en la Tabla 2.

Tabla 2: Niveles de hemoglobina y porcentaje de adecuación de ingesta de proteínas de alto valor biológico y hierro dietario por trimestre de gestación - 2018. Frecuencias y porcentajes.

\begin{tabular}{|c|c|c|c|c|c|c|}
\hline \multirow{3}{*}{ Variables } & \multicolumn{4}{|c|}{ Trimestre de gestación } & \multirow{2}{*}{\multicolumn{2}{|c|}{ Total }} \\
\hline & \multicolumn{2}{|c|}{ II } & \multicolumn{2}{|c|}{ III } & & \\
\hline & $\mathbf{n}$ & $\%$ & $\mathbf{n}$ & $\%$ & $\mathbf{n}$ & $\%$ \\
\hline \multicolumn{7}{|c|}{ Niveles de hemoglobina } \\
\hline Anemia moderada & 2 & 4,1 & 9 & 16,4 & 11 & 10,6 \\
\hline Anemia leve & 10 & 20,4 & 15 & 27,3 & 25 & 24,0 \\
\hline Normal & 37 & 75,5 & 31 & 56,4 & 68 & 65,4 \\
\hline \multicolumn{7}{|c|}{$\begin{array}{c}\text { Ingesta de proteínas de alto valor } \\
\text { biológico }\end{array}$} \\
\hline Ingesta Deficiente & 19 & 38,8 & 31 & 56,4 & 50 & 48,1 \\
\hline Ingesta Normal & 30 & 61,2 & 24 & 43,6 & 54 & 51,9 \\
\hline \multicolumn{7}{|c|}{ Ingesta de hierro dietario } \\
\hline Ingesta Deficiente & 24 & 49,0 & 37 & 67,3 & 61 & 58,7 \\
\hline Ingesta Normal & 25 & 51,0 & 18 & 32,7 & 43 & 41,3 \\
\hline Total & 49 & 100,0 & 55 & 100,0 & 104 & 100,0 \\
\hline
\end{tabular}

En la Tabla 3 puede observarse que la media de los niveles de hemoglobina estuvo dentro de los rangos normales $(11,4 \pm 1,11$; IC $95 \%$ : 11,1-11,6); sin embargo, la media del porcentaje de adecuación de la ingesta de proteína de alto valor biológico $(85,9 \pm 13,83$; IC $95 \%$ : 83,2-88,6) y hierro dietario $(79,4 \pm 14,49 ; \quad$ IC $95 \%$ : 76,6-82,2) estuvieron por debajo de los límites considerados como normalidad (Ingesta normal: $\geq 90 \%$ ). 
Tabla 3: Niveles de hemoglobina $(\mathrm{g} / \mathrm{dl}$ ) y porcentaje de adecuación de la ingesta de proteína de alto valor biológico y hierro dietario en gestantes - 2018. Media, desviación estándar (DE) e intervalos de confianza al 95\% (IC 95\%).

\begin{tabular}{|c|c|c|c|}
\hline Variables & Media & (DE) & IC 95\% \\
\hline Niveles de hemoglobina & & & \\
\hline Anemia moderada & 9,4 & 0,35 & $9,2-9,7$ \\
\hline Anemia leve & 10,5 & 0,35 & $10,3-10,7$ \\
\hline Normal & 12,0 & 0,70 & $11,9-12,2$ \\
\hline Total & 11,4 & 1,11 & $11,1-11,6$ \\
\hline Ingesta de proteínas de alto valor biológico & & & \\
\hline Ingesta deficiente & 74,7 & 11,21 & $71,5-77,9$ \\
\hline Ingesta normal & 96,3 & 5,05 & $94,9-97,7$ \\
\hline Total & 85,9 & 13,83 & $83,2-88,6$ \\
\hline Ingesta de hierro dietario & & & \\
\hline Ingesta deficiente & 70,6 & 12,97 & $67,3-73,9$ \\
\hline Ingesta normal & 91,8 & 2,28 & $91,1-92,5$ \\
\hline Total & 79,4 & 14,49 & $76,6-82,2$ \\
\hline
\end{tabular}

Se realizó la regresión lineal bivariada, observándose un alto grado de correlación positiva y estadísticamente significativa entre la ingesta de proteína de alto valor biológico con los niveles de hemoglobina $\left(r=0,88 ; \quad r^{2}=0,77\right.$; $p<0,001$ ) (Figura 1). Estos resultados que también se observaron en el segundo y tercer trimestre de gestación, respectivamente.

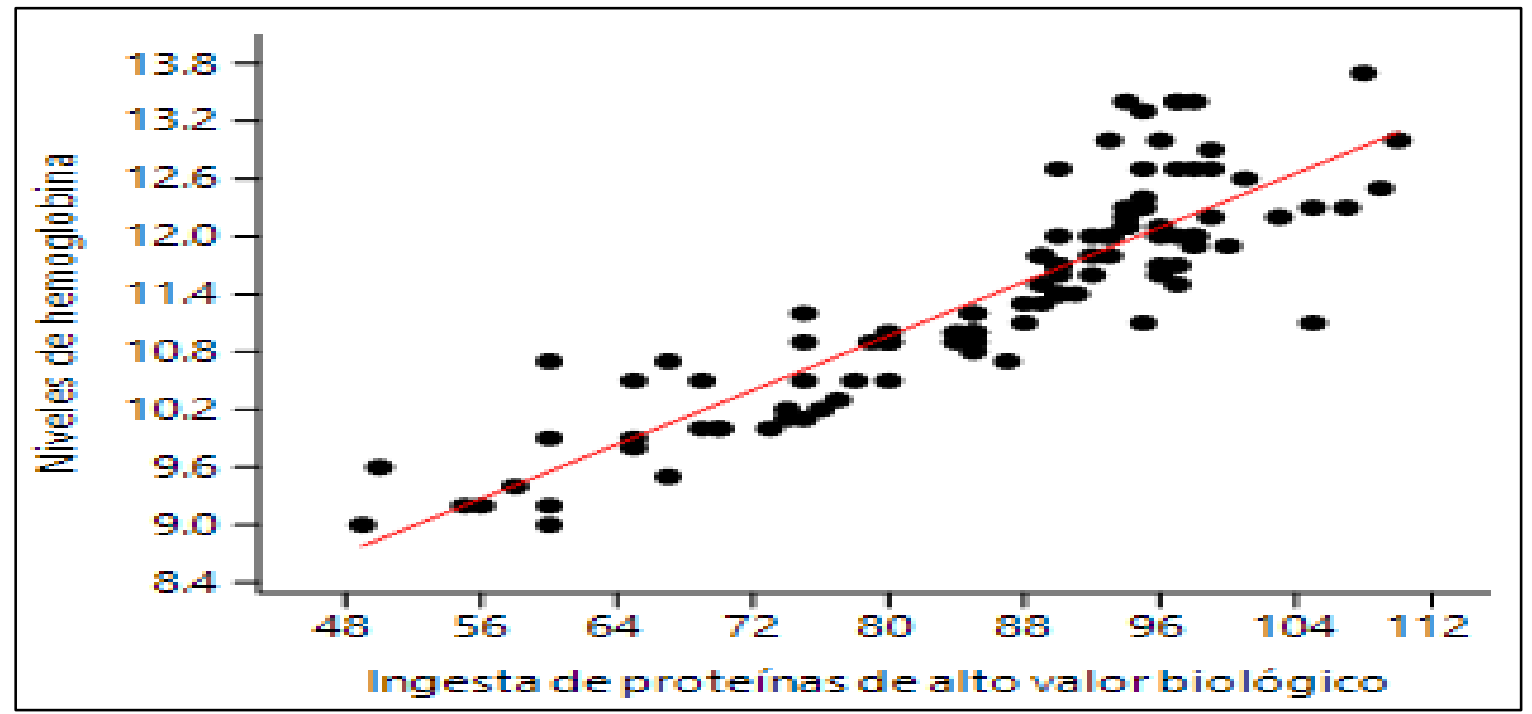

Figura 1: Correlación entre la ingesta de proteínas de alto valor biológico con los niveles de hemoglobina en gestantes - 2018.

La figura 2 muestra la regresión lineal bivariada entre la ingesta de hierro dietario con los niveles de hemoglobina, encontrándose un alto grado de correlación positiva y estadísticamente significativa entre estas dos variables ( $r>0,80$; $\left.r^{2}=0,69 ; p<0,001\right)$. Estos resultados también se evidenciaron en el segundo y tercer trimestre de gestación, respectivamente. 


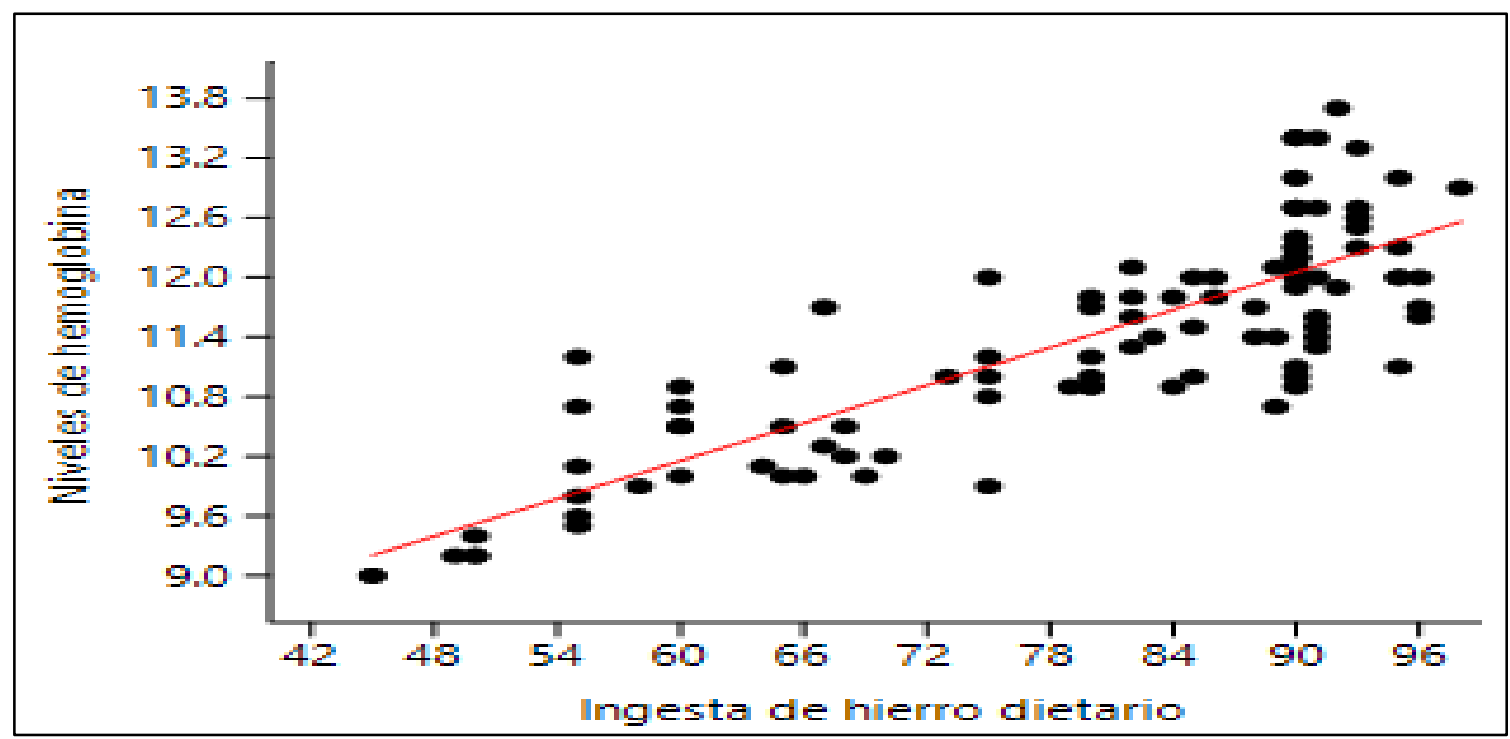

Figura 2: Correlación entre la ingesta de hierro dietario con los niveles de hemoglobina en gestantes - 2018.

Al realizar la regresión lineal múltiple entre la ingesta de proteína y hierro dietario con los niveles de hemoglobina se encontró una correlación positiva alta y con un elevado nivel de significancia entre estas tres variables de estudio $\left(r=0,88 ; r^{2}=0,78 ; p<0,001\right)$.

Por otro lado, el consumo energético promedio obtenido de la submuestra de gestantes fue de $2401,2 \pm 226,9 \mathrm{kcal}$ con un IC $95 \%$ de $2297,7-$ $2504,7 \mathrm{kcal}$.

\section{DISCUSIÓN}

Los resultados muestran que la ingesta habitual de las gestantes, adscritas al Hospital de Chincha, fue deficiente en proteínas de alto valor biológico y hierro dietario. Se atribuyen estas deficiencias nutricionales a una ingesta inferior a la recomendada de pescados, carnes y derivados, verduras y hortalizas; además, se observó que la ingesta de alimentos no tuvo una variabilidad adecuada. Estos aportes alimentarios insuficientes se reflejaron en los bajos niveles de hemoglobina en gestantes, encontrándose una alta prevalencia de anemia en esta población.

Según la OMS la prevalencia de anemia en gestantes a nivel mundial es cerca del $42 \%$. A nivel nacional el ENDES 2017 reporta 29,6\%3; el INS/CENAN 2015 encuentra $18,6 \%$ en el departamento de Ica, $18 \%$ en la provincia de Chincha y $19,6 \%$ en el distrito de Chincha Alta ${ }^{25}$. Este estudio encontró $34,6 \%$ de gestantes con anemia, distribuidos en $24,0 \%$ y $10,6 \%$ para anemia leve y moderada, respectivamente, resultados que están por encima de los reportados a nivel nacional, provincial y distrital. Estos hallazgos son muy relevantes en vista de que una gestante que carece de una alimentación adecuada, malnutrida, multigesta, con nivel socioeconómico bajo y con bajo nivel educativo esta propensa a padecer de anemia, así lo informa Caihuara et $a l^{9}$, y con ello presentar problemas o complicaciones durante el embarazo como prematuridad, riesgo de hemorragia, retraso del crecimiento intrauterino, mayor riesgo de transfusión sanguínea en el parto, bajo peso al nacer, mayor estancia hospitalaria, mayor riesgo de morbimortalidad materna, tal como lo informa Martínez et $a l^{2}$ y Rincón et $a l^{26}$. Munares et $a l^{10}$ encuentran que la prevalencia de anemia en gestantes es de $28 \%$ ( $25,1 \%$ de anemia leve y $2,6 \%$ de anemia moderada), siendo más prevalente en el tercer trimestre con 31\%, encontrándose cierta semejanza con los resultados encontrados. Sin embargo, al igual que los trabajos realizados por el ENDES 2017 y el INS/CENAN 2015, estos trabajos incluyen mujeres embarazadas de todas las edades lo que limitaría la comparación con los resultados de este estudio. Este trabajo no encontró relación significativa entre los niveles de hemoglobina y los indicadores como edad, estado civil, grado de instrucción y el número de gestación $(p>0,05)$, resultados que concuerdan con el realizado por San Gil et $a l^{27}$, sin embargo, se encontró relación significativa entre la ocupación de las gestantes con los niveles de hemoglobina $(p<0,05)$, las amas de casa fueron las más anémicas. Las gestantes que trabajaron tuvieron una menor afectación, probablemente porque esta 
condición les permitiría obtener un mayor ingreso familiar que repercutiría en la calidad y cantidad de los alimentos que se consumen así lo señala Caihuara et $a l^{9}$.

Por otro lado, se encontró que el $48,1 \%$ de gestantes presentaron ingesta deficiente de proteínas de alto valor biológico con una media de adecuación de $85,9 \%$, encontrándose la mayor cantidad de este déficit en el tercer trimestre. Las proteínas de origen animal (huevo, carnes, leche y derivados) contienen proteínas de alto valor bilógico que contienen aminoácidos indispensables, una disminución en su ingesta alteraría las funciones en el organismo como el acondicionamiento para la absorción, transporte y metabolismo del hierro y otros nutrientes; además, la biodisponibilidad del hierro (hemo) en los alimentos de origen animal es mayor y su absorción no se ve afectado por otros componentes de la alimentación ${ }^{6}$. Los agentes reductores por antonomasia son las proteínas de la dieta, esta condición mejoraría la absorción del hierro ${ }^{13}$; por lo tanto, al haber una ingesta deficiente de proteínas, sobre todo de alto valor biológico, los resultados reflejarían una disminución en los niveles de hemoglobina, produciendo anemia en las poblaciones más vulnerables como las gestantes, así lo manifiestan Espita y Orozco ${ }^{1}$. Al realizar la prueba bivariada se encontró un alto grado de correlación positiva y estadísticamente significativa entre la ingesta de proteínas de alto valor bilógico con los niveles de hemoglobina ( $r>0,88 ; p<0,001)$; por lo tanto, la ingesta de este macronutriente influye directamente sobre dichos niveles. Durante el embarazo es preciso consumir cantidades adecuadas de proteínas para cubrir necesidades derivadas del crecimiento del feto, de la placenta y de los tejidos maternos, dado que se estima que las proteínas depositadas en estos tejidos alcanzan cifras de $921 \mathrm{~g}$, es importante que la calidad de la proteína también sea adecuada ${ }^{28}$. No se ha encontrado estudios que relacionen la ingesta de proteínas de alto valor bilógico con los niveles de hemoglobina. Un estudio realizado por Quinteros et $a{ }^{29}$, muestra que las gestantes con anemia tuvieron adecuaciones bajas de energía, grasas y carbohidratos y un consumo elevado de proteínas; Irles et $a^{\beta 0}$, en su estudio sobre valor nutricional de la dieta en embarazadas sanas, obtiene resultados similares al anterior; ambos estudios no precisan el tipo de proteínas evaluadas, si es de alto o bajo valor bilógico, solo consideran la ingesta de proteína total. Coincidentemente con este trabajo, Piña et $a l^{31}$, en un trabajo realizado a 156 gestantes, concluye que existe un bajo consumo de alimentos de origen animal. De igual forma, Ferrer et $a^{32}$, en un estudio para identificar los patrones dietéticos durante el embarazo, encuentra una adecuación en la ingesta de proteínas de alto valor biológico en gestantes entre el 73 y $85 \%$, tomando como punto de corte el $70 \%$ de la ingesta como valor mínimo recomendado, valor distinto a lo señalado en esta propuesta que tuvo una adecuación mínima de $90 \%{ }^{33,34}$, aun así, en dicho estudio no se evalúa su relación con los niveles de hemoglobina; no obstante, el promedio de la adecuación alcanzada fue parecida a esta investigación.

Por otro lado, se encontró una ingesta deficiente de hierro dietario en más de la mitad de gestantes $(58,7 \%)$, con una media de adecuación de $79,4 \%$, siendo más deficiente en el tercer trimestre. Se realizó la prueba bivariada encontrándose un alto grado de correlación positiva y estadísticamente significativa entre la ingesta de hierro dietario con los niveles de hemoglobina $(r>0,80 ; p<0,001)$. Estos resultados coinciden con un estudio realizado por Montoya et $a^{35}$, el cual señala que existe una alta prevalencia de anémicas entre las mujeres embarazadas $(20,6 \%)$ producto de una baja ingesta de hierro y de los mayores requerimientos de hierro durante el embarazo. Irles et $\left.a\right|^{30}$, reporta que la ingesta deficiente de hierro dietario es el $70 \%$ en gestantes sanas, valor muy significativo y por encima de los valores registrados en el presente estudio. Saidman et $a^{\beta 6}$, en un trabajo realizado a 117 gestantes, encontró, como práctica común, una disminución en la ingesta del grupo de carnes, resultado similar a esta investigación. Este es un hallazgo preocupante porque la deficiencia en su consumo conlleva a un menor aporte de hierro, nutriente clave para la prevención de la anemia en gestantes y con ello evitar complicaciones en la madre, el feto y el recién nacido.

Los resultados de la regresión lineal múltiple entre la ingesta de proteína de alto valor biológico y hierro dietario con los niveles de hemoglobina en gestantes, demostraron que existe una alta correlación estadísticamente significativa y directamente proporcional entre estas variables $\left(r=0,88 ; r^{2}=0,78 ; p<0,001\right)$. El 
coeficiente de determinación $\left(r^{2}\right)$ señala que el $78 \%$ de la variabilidad de los niveles de hemoglobina pueden atribuirse a una relación lineal con la ingesta de proteínas de alto valor biológico y la ingesta de hierro dietario en gestantes, ello reafirma los hallazgos encontrados en esta investigación respecto de la relación de dependencia de estas variables.

Se debe señalar que la ingesta energética de la gestante fue adecuada, con una media de 2401 kcal, estando acorde con lo requerido para esta población tal como lo dispone el Ministerio de Salud 22; por tanto, los bajos niveles de hemoglobina no podrían ser atribuidos a este consumo.

Se precisa de estrategias de detección precoz de anemia, así como de atención y vigilancia nutricional que aseguren la intervención de forma adecuada y oportuna a gestantes con vulnerabilidad alimentaria y riesgos nutricionales. Asimismo, se sugiere a las autoridades del Seguro Social de Salud implementar programas que promuevan el consumo de una alimentación adecuada durante la etapa de gestación a fin de fomentar la ingesta de alimentos de origen animal y vegetal que proporcionen nutrientes que puedan prevenir la anemia. Una de las limitaciones importantes fue encontrar gestantes que ya habían sido atendidas en el primer trimestre de gestación, las cuales tuvieron que ser excluidas de la investigación a fin no generar sesgo en la información nutricional. Otra limitación es el diseño de estudio, al no ser experimental, limita establecer la relación causaefecto, solo establece una relación entre variables.

\section{CONCLUSIÓN}

Se concluye que los niveles de hemoglobina tienen relación directa y fuerte con la ingesta de proteínas de alto valor biológico y hierro dietario en gestantes. A partir de la presente investigación se espera que contribuya en comprender la necesidad de una ingesta adecuada y oportuna de proteínas y hierro en la dieta durante la gestación, y analizar sobre la problemática de estas respecto a sus factores inhibidores o que limitan su acceso en la dieta. Se espera que a partir de estos hallazgos se profundicen estudios relacionados a la digestibilidad de las proteínas y variabilidad en la absorción del hierro que permitan establecer su relación con los niveles de hemoglobina.

\section{DECLARACIÓN SOBRE CONFLICTOS DE INTERESES}

Los autores declaran no tener conflictos de intereses. Este estudio guarda correspondencia con una tesis realizada por uno de los autores en el año 2016, que no ha sido publicada en ninguna revista; no obstante, tanto el diseño metodológico como la población y muestra de estudio de la presente investigación son diferentes al de la tesis mencionada. Este trabajo fue autofinanciado.

\section{CONTRIBUCIÓN DE LOS AUTORES}

Primer autor: concepción y diseño del trabajo, recolección y obtención de resultados, análisis e interpretación de datos, elaboración y redacción del manuscrito, revisión crítica del manuscrito, aporte de pacientes de estudio, obtención de financiamiento, asesoría estadística, asesoría técnica.

Segundo autor: concepción y diseño del trabajo, análisis e interpretación de datos, revisión crítica del manuscrito, aprobación de su versión final.

\section{REFERENCIAS BIBLIOGRÁFICAS}

1. Espita F, Orozco L. Anemia en el embarazo, un problema de salud que puede prevenirse. Médicas UIS. 2013;26(3):45-50. Disponible en: https://revistas.uis.edu.co/index.php/revista medicasuis/article/view/3920/4267.

2. Martínez-Sánchez L, Jaramillo-Jaramillo L, Villegas-Álzate J, Álvarez-Hernández L, RuizMejía C. La anemia fisiológica frente a la patológica en el embarazo. Revista Cubana de Obstetricia y Ginecología. 2018; 44(2): 1-13. Disponible en: http://www.revginecobstetricia.sld.cu/index.p $\mathrm{hp} /$ gin/article/view/356.

3. Instituto Nacional de Estadística e Informática. Encuesta Demográfica y de Salud FamiliarENDES. Lima: INEI; 2017. Disponible en: https://www.inei.gob.pe/media/MenuRecursi vo/publicaciones_digitales/Est/Lib1525/index. html.

4. Véliz $N$, Peñaherrera $M$, Quiroz $M$, Mendoza $H$, Tonguino M. Prevención frente la presencia de anemia en el embarazo. Revista Científica Mundo de la Investigación y el Conocimiento. 
2019; 3(1): 971-996. Disponible en: https://dialnet.unirioja.es/servlet/articulo?co digo $=6796774$.

5. Pérez M, Basain J, Calderón G. Factores de riesgo del bajo peso al nacer. Acta Médica del Centro. 2018; 12(3): 369-382. Disponible en: http://www.revactamedicacentro.sld.cu/inde x.php/amc/article/view/885/1185.

6. Mahan K, Raymond J. Krause Dietoterapia. 14a. ed. Barcelona: Elsevier; 2017.

7. Servín M. Nutrición Básica y Aplicada. 2a. ed. Universidad Autónoma de México; 2013.

8. Kaufer M, Pérez A, Arroyo P. Nutriología Médica. 4a. ed. México: Editorial Médica Panamericana; 2015.

9. Caihuara $M$, Ortega $M$, Arteaga F. Anemia ferropénica, prevalencia en gestantes y puérperas. Centro de Salud Integral Santiago de Presto. Setiembre 2017 a febrero 2018. Archivos Bolivianos de Medicina. 2018; 29(97): 75-89. Disponible en: http://www.revistasbolivianas.org.bo/pdf/ab m/v29n97/v29n97_a08.pdf.

10. Munares O, Gómez G, Barboza J, Sánchez J. Niveles de hemoglobina en gestantes atendidas en establecimientos de salud del Ministerio de Salud del Perú 2011. Rev Peru Med Exp Salud Pública. 2012; 29(3): 329-336. http://www.scielo.org.pe/scielo.php?script=sc i_arttext\&pid=S172646342012000300006\&lng=es.

11. Mendoza L, Pérez B, Sánchez S. Estado nutricional de embarazadas en el último mes de gestación y su asociación con las medidas antropométricas de sus recién nacidos. Pediatr. (Asunción). 2010; 37(2): 91-96. Disponible en: https://dialnet.unirioja.es/servlet/articulo?co digo $=4800205$.

12. Institute of Medicine of The National Academies of Sciences. Dietary Reference Intakes: The Essential Guide to Nutrient Requirements. Washington, DC. 2006. Disponible en: https://www.nap.edu/read/11537/chapter/1.

13. Iglesias J, Tamez L, Reyes I. Anemia y embarazo, su relación con complicaciones maternas y perinatales. Medicina Universitaria. 2009; 11(43): 95-98.
14. Palacios J, Peña W. Prevalencia de anemia en gestantes de la ciudad de Huacho. Rev Peru Med Interna. 2014; 27(1): 6-10. Disponible en: https://pdfs.semanticscholar.org/de21/fa384a 0b94d2875d248aae6f39736b66b2ec.pdf.

15. Martínez O, Martínez E. Proteínas y péptidos en nutrición enteral. Nutrición Hospitalaria. 2006; 21(2): 1-14. Disponible en: http://scielo.isciii.es/scielo.php?script=sci_artt ext\&pid=S0212-16112006000500002\&Ing=es.

16. Suárez M, Kizlansky A, López L. Evaluación de la calidad de las proteínas en los alimentos calculándose el escore de aminoácidos corregido por digestibilidad. Nutr Hosp. 2006; 21(1): 47-51. Disponible en: http://scielo.isciii.es/scielo.php?script=sci_artt ext\&pid=S0212-16112006000100009\#back.

17. Vega L, Iñarritu M. Fundamentos de Nutrición y Dietética. 1a. ed. México: Pearson Educación de México; 2010.

18. Torresani M, Somoza M. Lineamientos para el cuidado nutricional. 3a. ed. Buenos Aires: Editorial Universitaria de Buenos Aires; 2011.

19. Aguilar S. Fórmulas para el cálculo de la muestra en investigación de salud. Salud en Tabasco. 2005; 11(1-2): 333-338. Disponible en:

https://www.redalyc.org/pdf/487/48711206.p df.

20. Ministerio de Salud Centro Nacional de Alimentación y Nutrición. Anemia en gestantes del Perú y provincias en comunidades nativas 2011. Lima: CENAN; 2012. Disponible en:https://web.ins.gob.pe/sites/default/files/ Archivos/cenan/van/informes/2013/12_Preva lencia\%20de\%20anemia\%20en\%20gestantes \%20v\%201_0_1.pdf.

21. Ministerio de Salud del Perú. Norma Técnica de Salud para el manejo terapéutico y preventivo de la anemia en niños, adolescentes, mujeres gestantes y puérperas. Lima: MINSA; 2017. Disponible en: http://bvs.minsa.gob.pe/local/MINSA/4190.p df.

22. Ministerio de Salud del Perú Centro Nacional de Alimentación y Nutrición. Requerimientos de Energía para la Población Peruana. Lima: CENAN; $2012 . \quad$ Disponible en: https://web.ins.gob.pe/sites/default/files/Arc 
hivos/cenan/deprydan/lamejorreceta/Requeri miento\%20de\%20energ\%C3\%ADa\%20para\%2 0la\%20poblaci\%C3\%B3n\%20peruana.pdf.

23. Pérez C, Aranceta J, Salvador G, VarelaMoreiras G. Métodos de frecuencia de consumo alimentario. Rev Esp Nutr Comunitaria. 2015; 21(1): 45-52. Disponible en:

http://www.renc.es/imagenes/auxiliar/files/R ENC2015supl1FFQ.pdf.

24. Hernández R. El Coeficiente de Proporción de Rangos: una alternativa para determinar la validez de contenido y el nivel de concordancia entre jueces en escalas likert. En: XXV Congreso Internacional de Psicología. San Juan, Puerto Rico: Universidad de los Andes; 1995. p.1-22.

25. Instituto Nacional de Salud Dirección Ejecutiva de Vigilancia Alimentaria y Nutricional. Estado Nutricional en niños y gestantes de los establecimientos de salud del Ministerio de Salud. Lima: DEVAN; 2015. Disponible en: https://web.ins.gob.pe/sites/default/files/Arc hivos/cenan/van/informes/1_ia2015.pdf.

26. Rincón D, González J, Urazán Y. Prevalencia y factores sociodemográficos asociados a anemia ferropénica en mujeres gestantes de Colombia (análisis secundario de la ENSIN 2010). Nutr. Hosp. 2019; 36(1): 87-95. DOI: 10.20960/nh.1895.

27. San Gil C, Villazán M, Ortega Y. Caracterización de la anemia durante el embarazo y algunos factores de riesgo asociados, en gestantes del Municipio Regla. Rev Cubana Med Gen Integr. 2014; 30(1): 71-80. Disponible en: http://scielo.sld.cu/scielo.php?script=sci_artte $x t \& p i d=S 0864-21252014000100007$.

28. Hernández A. Nutrición humana en el estado de salud. Tratado de Nutrición. 2a. ed. España: Panamericana; 2010.

29. Quinteros $Y$, Bastardo G, Angarita C, Paoli M, Sanz B, Rojas L, et al. Consumo de alimentos, factores socioeconómicos y anemia en mujeres gestantes. An Ven Nutr. 2012; 25(2): 64-72. Disponible en: https://www.analesdenutricion.org.ve/edicio nes/2012/2/art-3/.

30. Irles J, Iglesias $E$, Avilés $S$, Bernal E, Benito $P$, Moriones $L$, et al. Valor nutricional de la dieta en embarazadas sanas. Resultados de una ingesta dietética en gestantes. Nutrición Hospitalaria. 2003, 18(5): 248-252. Disponible en:

http://scielo.isciii.es/scielo.php?script=sci_artt ext\&pid=S0212-16112003000500004.

31. Piña F, La Torre L, Aylas W. Comportamiento, actitudes y prácticas de alimentación y nutrición en gestantes. Rev Per Obst Enf. 2007; 3(1): 17-21. Disponible en: https://www.aulavirtualusmp.pe/ojs/index.ph p/rpoe/article/view/539/411.

32. Ferrer C, García R, Mendez M, Romieul, Torrent $M$, Sunyer J. Determinantes sociales de los patrones dietéticos durante el embarazo. Gaceta Sanitaria. 2009; 23(1): 38$43 . \quad$ Disponible en: http://scielo.isciii.es/scielo.php?script=sci_artt ext\&pid=S0213-91112009000100008\&lng=es.

33. Couceiro C, Valdiviezo MS, Zimmer MC. Ingesta alimentaria y evaluación antropométrica de una misión aborigen de la provincia de Salta, República Argentina. Atención Primaria. 1997; 20(8): 421-424. Disponible en: https://www.elsevier.es/esrevista-atencion-primaria-27-articulo-ingestaalimentaria-evaluacion-antropometrica-una14675.

34. Díaz C, Valeria O, Biolley E. Ingesta dietaria de nutrientes críticos en embarazadas. Rev. chil. nutr. 2005; 32(3): 225-231. DOI: $10.4067 /$ S0717-75182005000300007.

35. Montoya J, Castelazo E, Valerio E, Velásquez G, Nava D, Escárcega J, et al. Opinión de un grupo de expertos en diagnóstico y tratamiento de la anemia en la mujer embarazada. Ginecol Obstet Mex. 2012; 80(9): 563-580. Disponible en: https://www.medigraphic.com/pdfs/ginobsm ex/gom-2012/gom129b.pdf.

36. Saidman $N$, Raele $M$, Basile $M$, Barreto $L$, Mackinonn $M$, Poy $M$, et al. Conocimientos, intereses y creencias sobre alimentación y nutrición en gestantes. DIAETA. 2012; 30(139): 18-27. Disponible en: http://www.scielo.org.ar/scielo.php?pid=S185 2-

$73372012000200004 \&$ script=sci_arttext $\&$ tIng =en 